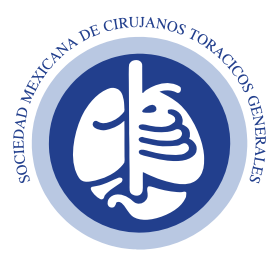

Vol. 1, Núm. 3

Septiembre-Diciembre 2020 pp 95-100

\title{
Tratamiento de hernia diafragmática traumática. Presentación de caso
}

\section{Treatment of traumatic diaphragmatic hernia. Case presentation}

\author{
Alan Barker-Antonio, * Mar Lisette Carmona-Pavón, ${ }^{\ddagger}$ \\ Roberto Armando García-Manzano, * Ediel Osvaldo Dávila-Ruíz, * \\ Kenia Escamilla-Orzuna, ${ }^{*}$ Dolores Isidoro-Hernández*
}

Palabras clave:

Hernia diafragmática

traumática, reparación

diafragmática,

trauma torácico.

Keywords:

Traumatic diaphragmatic hernia, diaphragmatic plication, thoracic trauma.

\footnotetext{
* Departamento de Cirugía General.

‡ Departamento de

Cirugía Cardiotorácica.
}

Hospital Regional de Alta Especialidad de Oaxaca. Oaxaca, México.

Recibido: 14/10/2020

Aceptado: 06/01/2021

Correspondencia:

Dr. Alan Barker-Antonio

Aldama s/n, Col. San Bartolo

Coyotepec, 71256,

Oaxaca, México.

Teléfono: 33 3444-1002

E-mail: drbarker91@ gmail.com

\begin{abstract}
RESUMEN
Introducción: La hernia diafragmática es una patología inusual, casi siempre asintomática, cuya frecuencia se encuentra en 0.2-1 por 1,000 adultos en las grandes series radiológicas. La plicatura diafragmática es el tratamiento más aceptado para la hernia diafragmática sintomática en adultos. Objetivo: Describir el caso de un paciente con hernia diafragmática secundaria a trauma de tórax, su estudio y resolución quirúrgica. Presentación de caso: Paciente masculino de 60 años de edad con antecedente de choque automovilístico hace 20 años, quien inicia su padecimiento con la presencia de disnea progresiva, la cual se exacerba los últimos tres meses previos a su internamiento, acompañado de dolor abdominal en hipocondrio derecho e izquierdo. Cursa con cuadro de oclusión intestinal, por lo que es hospitalizado; mediante estudios de imagen se demuestra disminución del parénquima pulmonar izquierdo por presencia de burbuja aparentemente hidroaérea gástrica. Se ingresa a tiempo quirúrgico donde se realiza laparotomía y toracotomía lateral anterior izquierda realizando plicatura diafragmática y colocación de malla. Conclusión: La hernia diafragmática es una patología muy infrecuente en nuestro medio; además, existe poca difusión para su manejo. La plicatura diafragmática y reforzamiento con malla de polipropileno es un procedimiento que se puede realizar en estos casos con buenos resultados.
\end{abstract}

\section{ABSTRACT}

Introduction: Diaphragmatic hernia is an unusual condition, almost always asymptomatic, whose frequency is $0.2-1$ per 1,000 adults in large radiological series. Diaphragmatic plication is the most widely accepted treatment for symptomatic diaphragmatic hernia in adults. Objective: To describe the case of a patient with a diaphragmatic hernia secondary to chest trauma, its study and surgical resolution. Case presentation: A 60-year-old male patient with a history of automobile shock 20 years ago who started his condition with the presence of progressive dyspnea, which exacerbated in the last three months prior to hospitalization, accompanied by abdominal pain in the right and left upper quadrant. He has a bowel occlusion picture and is therefore hospitalized. Imaging studies are performed where the chest X-ray shows a decrease in the left lung parenchyma due to the presence of an apparently gastric hydro aerial bubble. He enters the surgical time where a left anterior laparotomy and thoracotomy is performed, performing diaphragmatic plication and mesh placement. Conclusion: Diaphragmatic hernia is a very rare pathology in our environment, and there is little diffusion for its management. Diaphragmatic plication and reinforcement with polypropylene mesh is a procedure that can be performed in these cases with good results.

Citar como: Barker-Antonio A, Carmona-Pavón ML, García-Manzano RA, Dávila-Ruíz EO, Escamilla-Orzuna K, IsidoroHernández D. Tratamiento de hernia diafragmática traumática. Presentación de caso. Rev Mex Cir Torac Gen. 2020; 1(3); 95-100. https://dx.doi.org/10.35366/101478 


\section{INTRODUCCIÓN}

La hernia diafragmática surge como resultado del movimiento de los órganos abdominales hacia el tórax a través de un defecto del diafragma resultante de varios tipos de lesiones, que a menudo representan un desafío diagnóstico y terapéutico. La hernia diafragmática traumática es una enfermedad infrecuente, se estima que se presenta de $2.5-5 \%$ de los casos de traumatismo abdominal cerrado y $1.5 \%$ de los casos de trauma torácico cerrado, ${ }^{1}$ 15\% de los pacientes con traumatismos penetrantes de abdomen tienen afectación diafragmática, que en $50 \%$ de los casos pasan inadvertidas. $^{1}$ El trauma diafragmático rara vez es aislado, la mayoría de las veces se acompañan de otras lesiones toracoabdominales, cerebrales o musculoesqueléticas, estas comorbilidades más graves son responsables del mal pronóstico y aumento de la mortalidad, se estima que la mortalidad asociada con el trauma diafragmático varía entre 4.3 y $37 \% .^{2}$

El principal mecanismo de lesión en el trauma diafragmático son lesiones contusas en 75\% de los casos, las más representativas son accidentes automovilísticos de alta velocidad o secundario a una caída de gran altura, en las lesiones penetrantes los principales mecanismos son heridas por arma blanca y heridas por arma de fuego, con una incidencia variable, dependiendo de las condiciones socioeconómicas regionales. ${ }^{3} \mathrm{El}$ mecanismo fisiopatológico que determina la lesión diafragmática es el aumento repentino de la presión abdominal, lo que genera una presión excesiva sobre el diafragma y produce lesiones en el centro de la cúpula diafragmática, estas lesiones tienen como característica que presentan un tamaño que excede los $10 \mathrm{~cm}$, tiene una forma redondeada o en forma de hendidura con bordes que se asemejan a un anillo fibroso; ${ }^{4} \sin$ embargo, también se pueden presentar avulsiones de las inserciones musculares en pacientes que sufren impactos laterales. ${ }^{5}$

En trauma cerrado las lesiones del lado izquierdo son más comunes (75\%) en comparación con las lesiones del lado derecho (15\%), debido a la falta de acción protectora del hígado y a que las lesiones del lado derecho con mayor frecuencia permanecen sin ser reconocidas. En el trauma penetrante el daño al diafragma generalmente es del lado izquierdo debido al hecho de que la persona que lastima a menudo es diestra; mientras que con heridas de bala el número de lesiones del lado derecho y del lado izquierdo es aproximadamente el mismo, como característica en estas lesiones el defecto del diafragma a menudo no excede los $2 \mathrm{~cm} .{ }^{6}$ Las vísceras abdominales que con mayor frecuencia pueden sufrir herniación son el estómago y el intestino grueso. Las manifestaciones clínicas de las hernias diafragmáticas traumáticas son muy diversas y son el resultado de la isquemia y obstrucción de las vísceras herniadas, lo cual conduce a trastornos cardiopulmonares o gastrointestinales, los cuales, si son diagnosticados a tiempo, pueden disminuir drásticamente la mortalidad de esta patología. ${ }^{6}$

El método diagnóstico estándar para esta patología es la radiografía de tórax, la cual tiene una sensibilidad de $46 \%$ para lesiones del lado izquierdo y de $17 \%$ para lesiones del lado derecho en la fase aguda; sin embargo, el desarrollo de nuevas técnicas de imagen, como la tomografía computarizada, nos ha permitido un aumento en la tasa de diagnóstico temprano y correcto, la sensibilidad en el período agudo de la lesión fue de $78 \%$ para las lesiones del lado izquierdo del diafragma y de $50 \%$ en el caso de las lesiones del lado

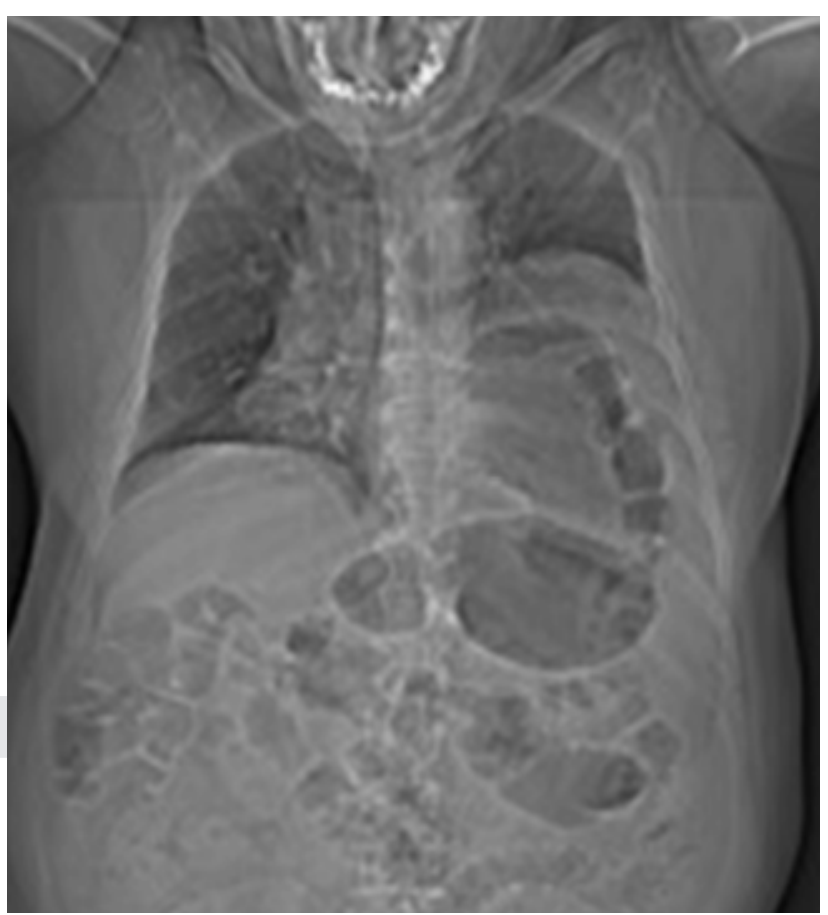

Figura 1: Elevación de hemidiafragma izquierdo con disminución del parénquima pulmonar. 

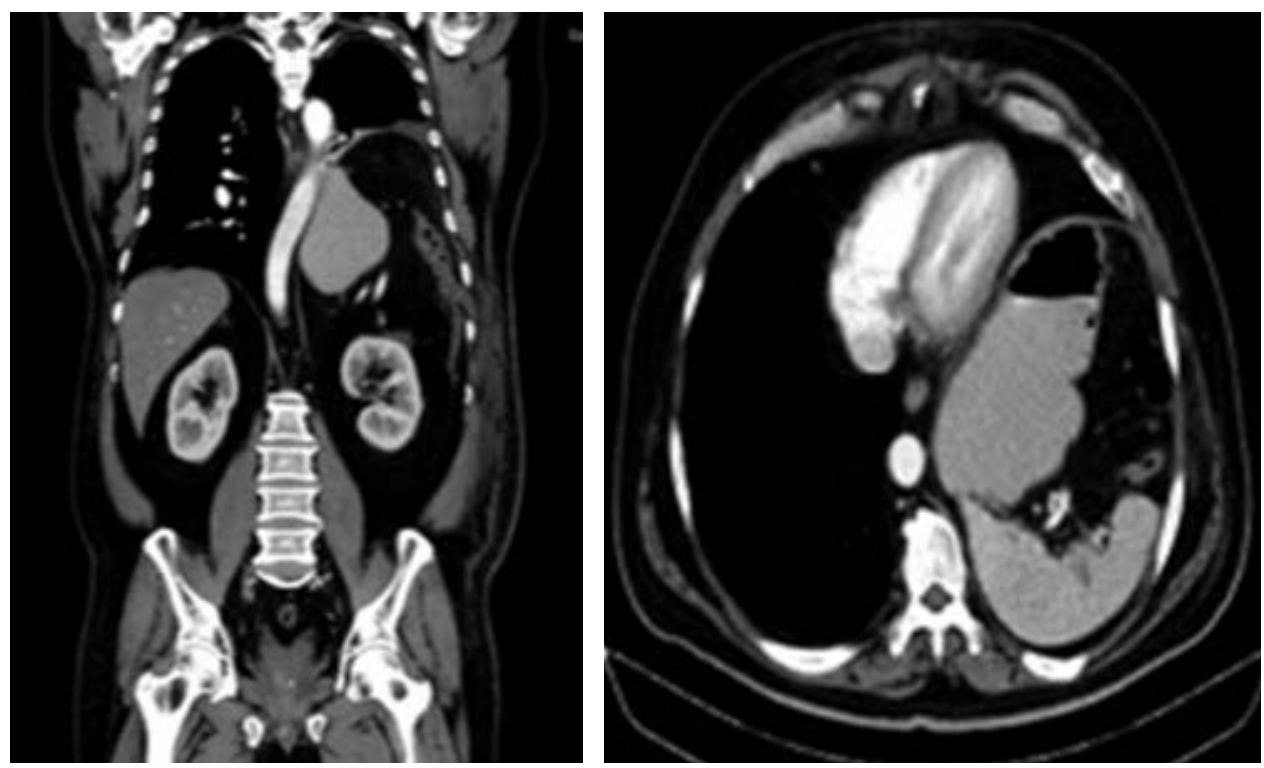

Figura 2:

Tomografía axial computarizada de tórax con evidencia de estómago y bazo en hemitórax izquierdo.

derecho, las ventajas de este estudio es que permite visualizar directamente el defecto del diafragma, su ubicación y tamaño, así como detallar los órganos que han ingresado a la cavidad pleural. ${ }^{7}$

\section{PRESENTACIÓN DEL CASO}

Paciente masculino de 60 años de edad, sin antecedentes personales patológicos de importancia, con antecedente de choque automovilístico hace 20 años que no ameritó hospitalización; inició su padecimiento actual con la presencia de disnea progresiva, la cual exacerbó los últimos tres meses previos a su internamiento, acompañado de dolor abdominal en hipocondrio derecho e izquierdo.

Cursó con distensión abdominal y ausencia de evacuaciones, se realizó radiografía de tórax (Figura 1), la cual mostró disminución del parénquima pulmonar izquierdo por presencia de burbuja aparentemente hidroaérea gástrica; ante estos hallazgos se decidió realizar tomografía de tórax (Figura 2) que reportó nivel hidroaéreo arriba del límite diafragmático izquierdo haciendo un cuello de botella en la continuación del tracto digestivo, además de visualizarse bazo con engrosamiento cicatrizal en la periferia hacia pared torácica.

Se ingresó a tiempo quirúrgico donde se realizó abordaje de la hernia diafragmática mediante toracotomía lateral izquierda en la que se observó saco herniario (Figura 3), el cual no se logró reducir por esta vía, por lo que fue necesario realizar un abordaje abdominal mediante laparotomía para poder realizar la reducción del contenido herniario (Figura 4), la cual fue difícil debido a la presencia de adherencias de epiplón que dificultaron el descenso del contenido a la cavidad abdominal y en el cual se encontraban el bazo, estómago, epiplón y colon transverso. Una vez lograda la reducción de la hernia se identificó un anillo de $10 \mathrm{~cm}$, al cual se le realizó un cierre primario mediante técnica de bolsa de tabaco en dos planos con material no absorbible (Prolene 1) (Figura 5); se

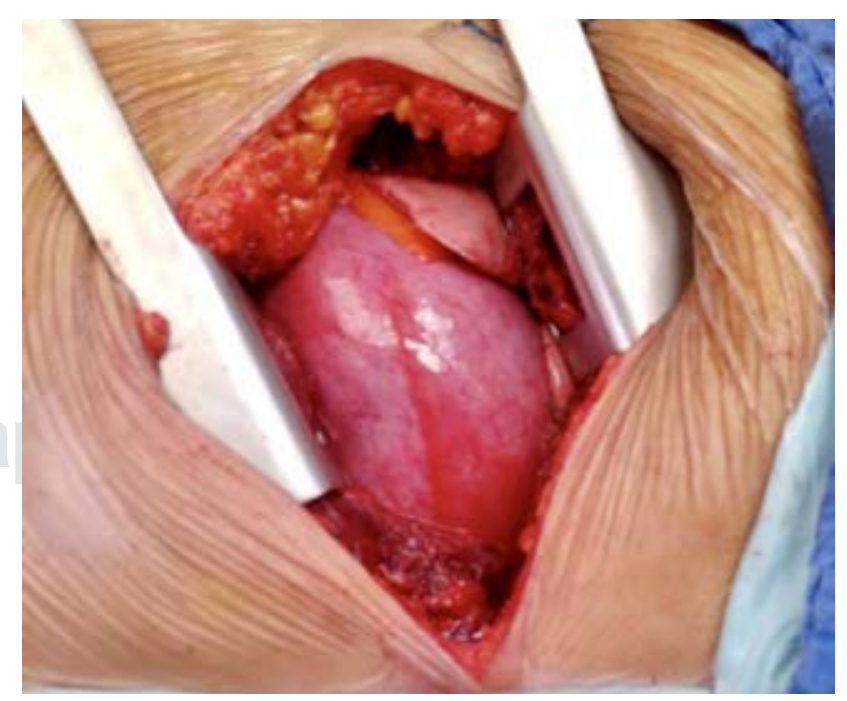

Figura 3: Hernia diafragmática. 


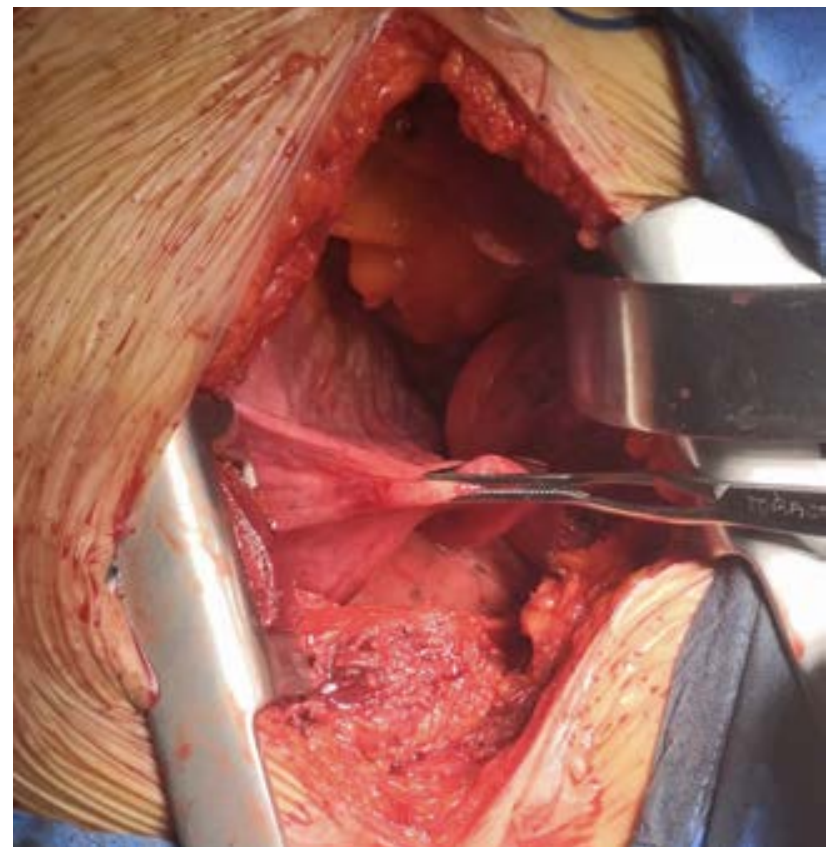

Figura 4: Hemidiafragma izquierdo después de reducir el contenido.

realizó fortalecimiento de la plastía con colocación de malla de polipropileno (Figura 6), la cual se fijó a cúpula diafragmática. Cursó con adecuada evolución posoperatoria, se inició la vía oral a las 24 horas con buena tolerancia; se retiró la sonda endopleural al cuarto día de posoperado, tuvo un gasto total en esos días de $500 \mathrm{ml}$ de contenido serohemático y fue egresado al quinto día del procedimiento. En el seguimiento a los seis meses de procedimiento el paciente se encuentra sin complicaciones.

\section{DISCUSIÓN}

Las hernias diafragmáticas representan un verdadero desafío clínico y quirúrgico; se requiere un alto índice de sospecha y el uso adecuado de las imágenes diagnósticas que permitan la resolución del problema durante la misma hospitalización del paciente para evitar complicaciones a largo plazo, las cuales resultan catastróficas. Independientemente de la fase de inicio o la presencia de obstrucción o isquemia, la cirugía es el único tratamiento para la resolución de esta patología; sin embargo, existe controversia acerca de las ventajas y desventajas de los diferentes enfoques quirúrgicos, ya sea abierto o mínimamente invasivo.
Una revisión sistemática reciente que incluyó a 2,023 pacientes mostró que, en la fase aguda, un abordaje abdominal es más recomendable, mientras que en la fase crónica el abordaje torácico es más frecuente, el requisito de abrir la segunda cavidad es similar en ambos enfoques. ${ }^{8}$ El abordaje abierto es utilizado en el tratamiento de lesiones diafragmáticas en fase aguda y se ha demostrado que se repara con éxito en la mayoría de los casos; no obstante, la introducción de la cirugía mínimamente invasiva ha permitido que este enfoque sea utilizado como primera instancia en aquellos pacientes en quienes por métodos de imagen se ha evidenciado que no existen otras lesiones asociadas; aunado a que la laparoscopia diagnóstica puede excluir otras lesiones intraabdominales, evitando así la laparotomía exploratoria innecesaria y minimizando el tiempo de estancia intrahospitalaria, los costos, y logrando una recuperación más rápida y con mejor cosmética a largo plazo. ${ }^{1}$ Existen varios reportes, con series pequeñas, de corrección de lesiones del diafragma por laparoscopia, con resultados satisfactorios y porcentajes pequeños de conversión a laparotomía. ${ }^{9}$

En este sentido está claro que las técnicas quirúrgicas mínimamente invasivas son el estándar

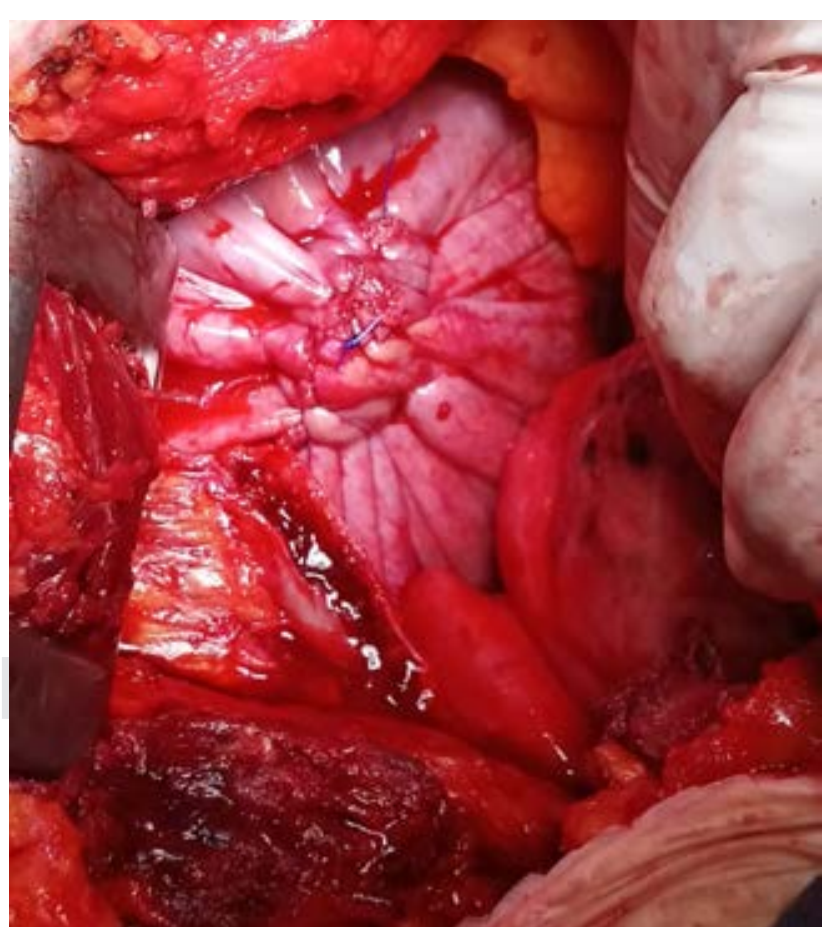

Figura 5: Plicatura diafragmática. 


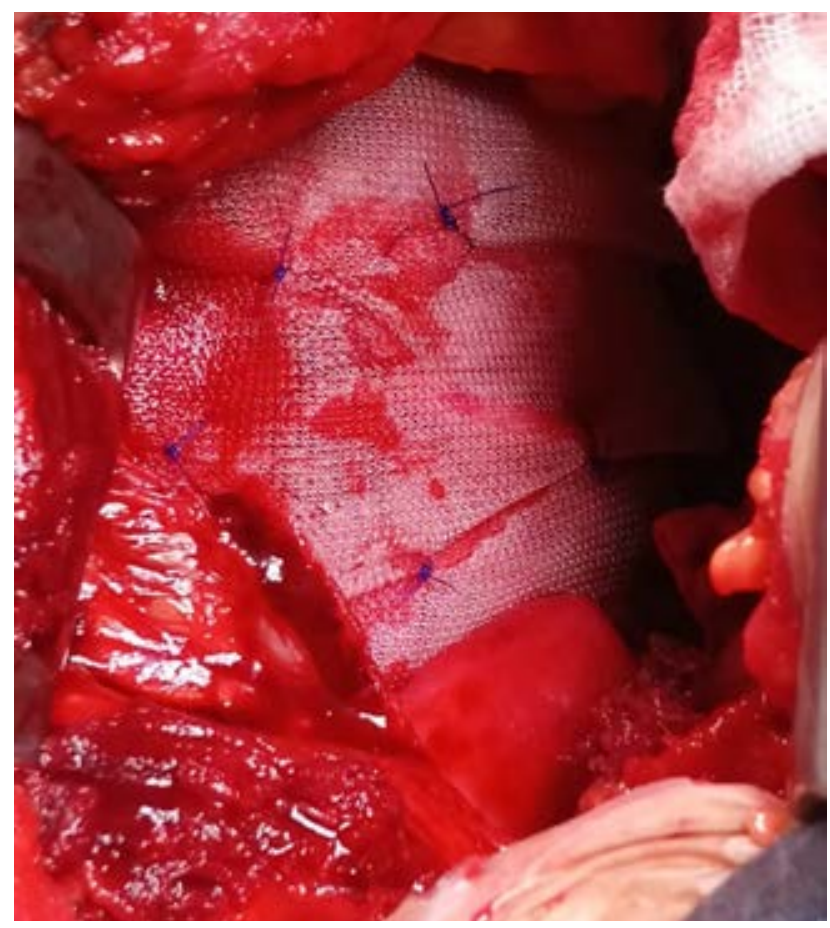

Figura 6: Colocación de malla de polipropileno para el reforzamiento.

de diagnóstico e, incluso, pueden llegar a serlo de tratamiento; sin embargo, es necesario tener el entrenamiento y la experiencia para realizar estos procedimientos por esta vía. En nuestro caso no se contaba con ello para llevar acabo la reparación mediante técnicas mínimamente invasivas, además de que el paciente presentaba datos de encarcelamiento como complicación de la hernia diafragmática, por lo que se optó por realizar el abordaje quirúrgico de manera abierta.

La elección del abordaje se debe individualizar con base en el órgano lesionado, ya sea torácico o abdominal, y depende de las preferencias del cirujano, así como de su experiencia quirúrgica. ${ }^{1}$ La mayor parte de las hernias diafragmáticas traumáticas están constituidas por un solo órgano, el estómago con mayor frecuencia, seguido por colon, bazo, intestino delgado y epiplón; según el tamaño del defecto pueden existir dos o más órganos como en este caso. ${ }^{10}$

Se ha descrito que la plicatura diafragmática es muy poco realizada en adultos, siendo más aceptada en la población pediátrica; sin embargo, existen pocas publicaciones con una casuística limitada de plicatura dia- fragmática en adultos, en todas se muestran resultados satisfactorios y leves complicaciones que se reducen al realizar técnicas mínimamente invasivas. ${ }^{11}$ El trabajo de Freeman y colaboradores ${ }^{12}$ es un estudio de cohortes que compara siete pacientes con hernia diafragmática sin operar con otro grupo de pacientes operados de plicatura diafragmática de 11 casos por toracotomía y 30 casos por cirugía toracoscópica asistida por video (VATS); sin embargo, por el número pequeño de casos y el diseño del estudio no es posible que se considere este trabajo como un nivel de evidencia IIB; el resto de publicaciones relacionados con este tema se limitan a series de casos.

Está descrito en la literatura mundial que la técnica quirúrgica de reparación va encaminada a dar mayor soporte al músculo diafragmático, ya sea con plicatura muscular solamente o plicatura más la adición de material protésico en el sitio de sutura con mallas sintéticas de polipropileno, Dual Mesh (Goretex) o Mersilene. ${ }^{13}$

Dentro de las principales complicaciones posoperatorias encontramos neumonía, atelectasia, infección del tracto urinario, infección de la herida quirúrgica, empiema, íleo adinámico, sepsis, parálisis diafragmática, entre otras; sin embargo, no es posible determinar cuál abordaje conduce a la mayor cantidad de complicaciones. $^{14}$

\section{CONCLUSIÓN}

La hernia diafragmática es una patología muy infrecuente en nuestro medio; además, existe poca difusión para su manejo. Hay que sospechar de esta patología en pacientes con antecedente de trauma torácico y que presentan disnea como única manifestación clínica. La plicatura diafragmática y reforzamiento con malla de polipropileno es un procedimiento que se puede realizar en estos casos con buenos resultados.

\section{REFERENCIAS}

1. Singh D, Aggarwal S, Vyas S. Laparoscopic repair of recurrent traumatic diaphragmatic hernia. J Minim Access Surg. 2019; 16(2): 166-168.

2. Gu P, Lu Y, Li X, Lin X. Acute and chronic traumatic diaphragmatic hernia: 10 years' experience. PLoS One. 2019; 14(12): e0226364.

3. Porojan VA, David OI, Coman IS, Coman EV, Draghici DA, Popescu $C$ et al. Traumatic diaphragmatic lesions - considerations over a series of 15 consecutive cases. Chirurgia (Bucur). 2019; 114(1): 73-82. 
4. Parshin VD, Khetagurov MA. Diaphragmatic hernia in elective thoracic surgery. Khirurgiia (Mosk). 2018; (8): 36-45.

5. Scumpia AJ, Aronovich DA, Roman L, Vasudevan V, Shadis RM, Lynn M. Diaphragmatic rupture secondary to blunt thoracic trauma. West J Emerg Med. 2013; 14(5): 435-436.

6. Wakai S, Otsuka H, Aoki H, Yamagiwa T, Nakagawa Y, Inokuchi S. A case of incarcerated and perforated stomach in delayed traumatic diaphragmatic hernia. Tokai J Exp Clin Med. 2017; 42(2): 85-88.

7. Bergin D, Ennis R, Keogh C, Fenlon HM, Murray JG. The "dependent viscera" sign in CT diagnosis of blunt traumatic diaphragmatic rupture. AJR Am J Roentgenol. 2001; 177(5): 1137-1140.

8. Siow SL, Wong CM, Hardin M, Sohail M. Successful laparoscopic management of combined traumatic diaphragmatic rupture and abdominal wall hernia: a case report. J Med Case Rep. 2016; 10: 11.

9. Zerey M, Heniford BT, Sing Rf. Laparoscopic repair of traumatic diaphragmatic hernia. Oper Tech Otolayngol Head Neck Sur. 2006; 8: 27-33.
10. Chughtai T, Ali S, Sharkey P, Lins M, Rizoli S. Update on managing diaphragmatic rupture in blunt trauma: a review of 208 consecutive cases. Can J Surg. 2009; 52(3): 177-181.

11. Gazala S, Hunt I, Bédard EL. Diaphragmatic plication offers functional improvement in dyspnoea and better pulmonary function with low morbidity. Interact Cardiovasc Thorac Surg. 2012; 15 (3): 505-508.

12. Freeman RK, Van Woerkom J, Vyverberg A, Ascioti AJ. Long-term follow-up of the functional and physiologic results of diaphragm plication in adults with unilateral diaphragm paralysis. Ann Thorac Surg. 2009; 88(4): 1112-1117.

13. O'Farril AR, Molina RD. Diagnóstico y tratamiento de la eventración diafragmática. Cir Cen. 2007; 29(3): 225-229.

14. Silva GP, Cataneo DC, Cataneo AJM. Thoracotomy compared to laparotomy in the traumatic diaphragmatic hernia. Systematic review and proportional methanalysis. Acta Cir Bras. 2018; 33(1): 49-66. 